\title{
Complicated Meconium Ileus: Case Report with Review of Literature
}

\author{
Dr. Anand ${ }^{1}$, Dr. Sreeramulu .P. $\mathbf{N}^{2}$, Dr. Rahul Singh $\mathbf{R}^{3}$ \\ ${ }^{1}$ Associate Professor, Department of Pediatric Surgery \\ ${ }^{2}$ Professor, Department of General Surgery \\ ${ }^{3}$ Post Graduate in Surgery, SDUMC. Kolar
}

\begin{abstract}
Meconium ileus (MI) is a unique form of neonatal obstruction almost always associated with Cystic Fibrosis. Uncomplicated cases respond well to nonsurgical management.However, few patients develop complications in the postnatal period, requiring emergency operation.A two day old newborn presented with clinical features of low intestinal obstruction suspected to be due to meconium ileus.After resuscitation, a trial of conservative treatment, with Mucomist enemas, was attempted without success. Exploratory laparotomy revealed typical MI with midileal volvulus. Resection of the gangrenous, volvulated ileum with manual evacuation of meconium, both proximally and distally, followed by end to end anastomosis was done. Patient made uneventful recovery. Followup tests did not reveal cystic fibrosis, the growth and development of the baby has been uneventful.
\end{abstract}

Keywords: Meconium ileus, Volvulus, Gangrene, Cystic Fibrosis

\section{Introduction}

Meconium ileus (MI) is defined as an obstruction caused by inspissated meconium, at the level of the terminal ileum. ${ }^{1}$ It is a unique form of neonatal small bowel obstruction with or without association of cystic fibrosis (CF). Nearly $80 \%$ cases of Meconium Ileus is associated with Cystic Fibrosis. Only around $10-20 \%$ of cases have been reported to have isolated meconium ileus without any features of CF. However MI complicated by perforation,Atresia, volvulus,gangrene etc.,of the affected ileum, requires emergency surgical intervention, with majority undergoinga two staged procedure. ${ }^{2}$ The Prognosis of those babies presenting with uncomplicated MI is generally good and a trial of conservative management can be given.

\section{Case Report}

A two day old, term male baby,weighing $2.6 \mathrm{~kg}$, presented with clinical features of gross abdominal distension, bilious vomiting and non-passage of meconium, of 1 day duration. General physical examination, revealed a lethargic and ill looking baby with gross abdominal distension\&moderate respiratory distress. There was no erythema or edema of the abdominal wall. A vague, ill-defined mass was felt in mid abdomen. Bowel sounds were present. He was febrile with temperature of $99{ }^{\circ} \mathrm{F}$; respiratory rate $48 / \mathrm{pm}$, and pulse $143 \mathrm{bpm}$.

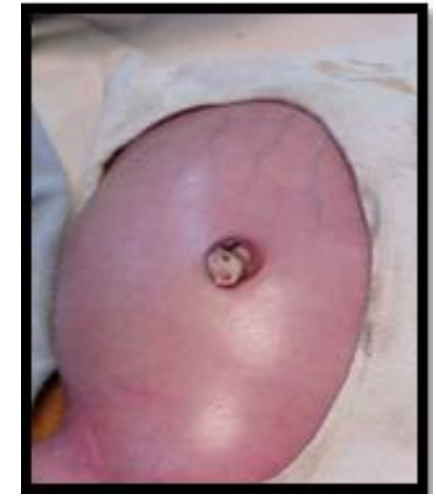

Figure 1: Clinical Photograph Showing Distended Abdomen

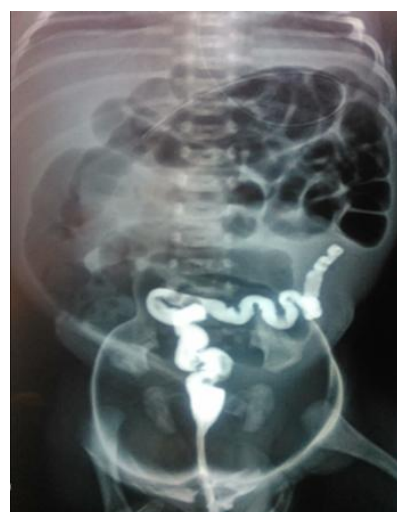

Figure 2: Contrast Enema showing Micro Colon showing gaseous distension 


\section{International Journal of Science and Research (IJSR) \\ ISSN (Online): 2319-7064}

Index Copernicus Value (2015): 78.96 | Impact Factor (2015): 6.391

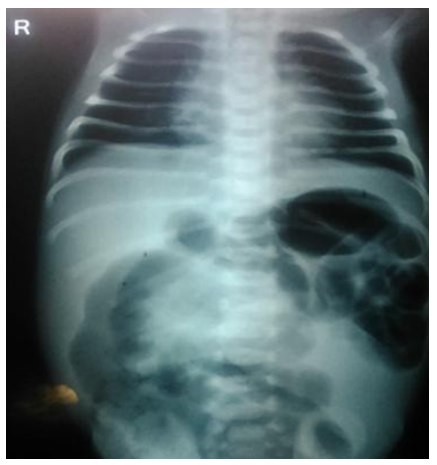

Figure 3: Erect x-ray Abdomen Absent fluid levels, soft tissue mass

X-ray abdomen showed, dilated bowel loops of varying sizes without fluid levels, or evidence of perforation.Contrast $\mathrm{x}$-rays, with Omnipaque enema showed micro colon, with filling defects, due to meconium beads. The newborn was resuscitated with nasogastric aspiration, IV fluids/ electrolytes and antibiotics and takenup for surgery. Exploration revealed, typical MI findings.Of inspissated meconium pellets in distal ileum and colon,marked dilatation of proximalileum, which was filled with thick, sticky meconium,volvulus of the mid ileal segment with signs of devitalisation and a near clean looking peritoneal cavity. Resection of the volvulatedileal segment, manual evacuation of abnormal meconium, assisted by Mucomist(NAcetyl cysteine) irrigation, followed by end to end anastomosis, was done. Patient made an uneventful recovery and was discharged in good condition. Two year follow up, revealed a healthy child, with no complaints of constipation, repeated chest infections or malabsorption.

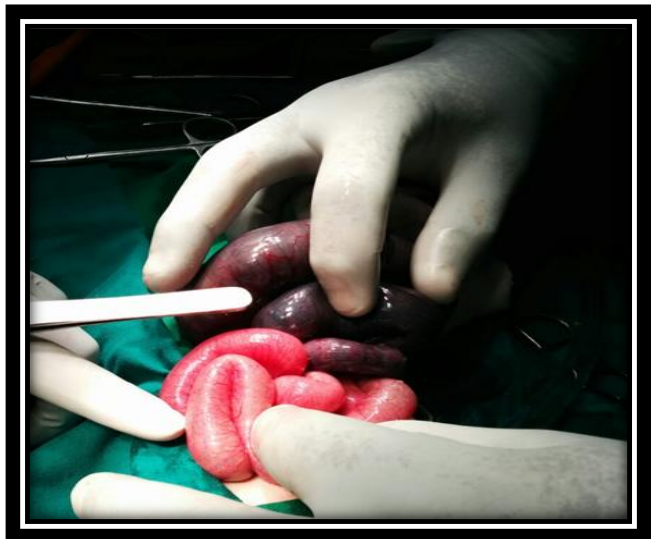

Figure 4: Gangrenous Bowel Segment

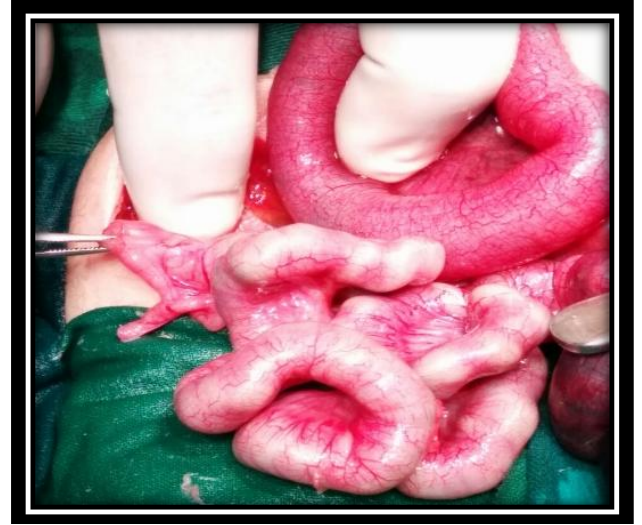

Figure 5: Distal Ileum loaded with Meconium Pellets, giving a beaded appearance

\section{Discussion}

Meconium is the earliest stool of a mammalian infant. Composed of materials ingested during intra uterine life and contains intestinal epithelial cells, lanugo hair, mucus, bile salts, bile pigments, amniotic fluid etc. It is dark green in colour, viscous and sticky, almost odourless and passed within 24 hours after birth.

Meconium ileus is a condition where in extremely viscid, protein rich, inspissated meconium, causing intraluminal obturator type of obstruction of distal ileum by thickly passed mucus plugs that resemble rabbit pellets. ${ }^{4}$ Nearly $80 \%$ cases of Meconium Ileus is associated with Cystic Fibrosis. More common in the Caucasians with equal incidence in both sexes. Only around 10- $20 \%$ of cases have been reported to have Isolated meconium ileus without any features of Cystic Fibrosis. ${ }^{5}$ Our case was among those $20 \%$ which is unusual with no signs of prematurity, no family history. Other causes of Meconium Ileus have also been studied like- Pancreatic aplasia, colon aganglionosis, Pancreatic ductal stenosis, ileocaecal atresia, familial conditions, prematurity.

Two types of Meconium Ileus are described Simple (uncomplicated)/Complicated.

Simple (uncomplicated) MI: baby at birth is normal, no abdominal distension but as time goes by develops abdominal distension, bilious vomiting, careful palpation of abdomen reveals a "doughy" abdomen with indentable masses (meconium clogged bowel).

Complicated Meconium Ileus: Baby is born with significant abdominal distension, respiratory distress with poor general condition. Examination reveals signs of meconium peritonitis/obstruction, ascites. Rectal wash with saline will expel a small amount of thick, viscid meconium. A mass palpable suggesting pseudo cyst, probably due to inutero bowel perforation. The common complications that are encountered are ileal atresia or stenosis, ileal perforation resulting in meconium peritonitis, volvulus with or without pseudo cyst formation.

On a plain radiograph, simple meconium ileus shows unevenly dilated bowel loops with or without air fluid levels due to increased viscosity of the meconium not allowing air interface with the fluid. Occasionally, has a mottled appearance on radiographs during the first 2 days of life, "bubbly" appearance of the distended intestinal loops (Neuhausser's Sign) particularly in the right lower quadrant. Contrast enema will show a micro colon (micro colon of disuse) involving the entire large bowel and may show impacted meconium pellets particularly in the right colon or in the distal ileum. ${ }^{7}$ Undue precautions should be taken to resuscitate the sick babies prior to subjecting them to any contrast studies. In a complicated meconium ileus radiographs will reveal signs of obstruction/ giant cysts, ascites and/or calcifications.

Prenatal ultrasound can help in the earlier diagnosis so that suitable precautions can be taken postnatally, findings include echogenic bowel, which can be dilated and thick 


\section{International Journal of Science and Research (IJSR) \\ ISSN (Online): 2319-7064}

Index Copernicus Value (2015): 78.96 | Impact Factor (2015): 6.391

walled, polyhydramnios, fetal ascites, peritoneal wall calcifications, intra-abdominal cysts. ${ }^{8}$ Postnatal findings include distended bowels with meconium (in simple MI), ascites, and giant intra-abdominal cysts (in complicated MI).

Meconium ileus is often the first sign of Cystic Fibrosis. The basic genetic abnormality in Cystic Fibrosis is mutation in CFTR (cystic fibrosis transmembrane regulator) gene located on 7q31 chromosome, F508 mutation, causing 3 base pair deletion where phenylalanine residue is removed at 508 position. Abnormal CFTR gene produces abnormal electrolyte content in the environment external to intestinal apical cells, which leads to reduced clearance of secretions from tubular structures lined by the affected epithelium. Chronic intestinal obstruction, abnormal respiratory tract, exocrine pancreas insufficiency, elevated sweat chloride levels are the features.

Meconium ileus (simple) should be differentiated from meconium plug syndrome, Hirschprung's Disease and bowel atresia. $^{9}$

Majority of these patients are usually sick and dehydrated with electrolyte disturbances. They need intensive resuscitation with IV fluids and antibiotics before subjecting them to invasive procedures or surgery. In simple MI nearly $68 \%$ of cases can be managed conservatively with enemas using Omnipaque, gastrograffin, under fluoroscopic control warm saline enemas containing $1 \% \mathrm{~N}$-acetyl Cysteine. Two failed attempts and operative intervention is needed. ${ }^{10}$ Surgery involves Enterotomy and evacuation of all the abnormal meconium assisted by local irrigation with Mucomist (1\% N Acetyl Cysteine) or Gastrograffin (in a fit patient). Other cases are managed by staged procedures like Mickulikz, Bishop Koop, and Santulli Blanc. Complicated MI are managed by laparotomy, resection followed by ileostomy. Intestinal continuity is established 6-8 weeks post operatively.

Prognosis is the best in isolated, uncomplicated cases without Cystic fibrosis. Patients with $\mathrm{CF}$ have chronic problems like repeated chest infections, pancreatic insufficiency with malnourishment, recurrent attacks of constipation and abdominal distension (meconium ileus equivalent), fertility disorders etc.

We present this case because of its rarity and to create an awareness that meconium ileus could be a reason for distal neonatal intestinal obstruction. Instead of a staged procedure this complicated MI was managed by a single staged resection and anastomosis due to reasonably good general condition and absence of overt peritonitis.

\section{Conclusion}

The presentation of simple meconium ileus with volvulus can be missed if not taken into consideration. Early surgical intervention is required to manage such a situation to avoid complications. Meconium ileus can be a reason for distal neonatal intestinal obstruction.

\section{References}

[1] Kayastha et al, Postnatal Volvulus In Meconium Ileus, APSP J Case Rep 2011; 2: 7

[2] Ziegler MM. Meconium Ileus. In: O’ Neil JA, Rowe MI, Grosfeld JL, Fonkalsrud EW, Coran AG. Editors. Pediatric Surgery, 6th ed, Philadelphia: mosby, 2006:1289-03.

[3] Estroff JA, Bromley B, Benacerraf BR. Fetal meconium peritonitis without sequelae. Pediatr Radiol1992; 22:277-8.

[4] P H Weller, J Williams. Journal of the Royal Society of Medicine Supplement No. 12 Volume $791986: 36-7$

[5] Johnson JA, Bush A, Buchdahl R. Does presenting with meconium ileus affect the prognosis of children with cystic fibrosis? PediatrPulmonol 2010 Oct; 45(10):9518.

[6] Murshed R, Spitz L, Kiely E, Drake D. Meconium ileus: a ten-year review of thirty-six patients. Eur J Pediatr Surg. 1997 Oct; 7 (5) : 275-7

[7] Kappler M, Griese M. Meconium ileus-it is time to act now! PediatrPulmonol. 2010 Oct; 45(10):949-50.

[8] Burge D, Drewett M. Meconium plug obstruction. PediatrSurg Int. 2004 Feb; 20(2):108-10.

[9] Copeland DR, St Peter SD, Sharp SW, Islam S, Cuenca A, Tolleson JS, Dassinger MS, Little DC, Jackson RJ, Kokoska ER, Smith SD. Diminishing role of contrast enema in simple meconium ileus. J Pediatr Surg. 2009 Nov; 44(11):2130-2.

[10] Gillis Da, GrantmyreEb. Can Med Assoc J. 1965 Jan 30; 92:225-7. 